\title{
Synthesis of Correct-by-construction Control Protocols for Hybrid Systems Using Partial State Information
}

\author{
Oscar Mickelin ${ }^{1}$, Necmiye Ozay $^{2}$ and Richard M. Murray ${ }^{3}$
}

\begin{abstract}
This paper considers the problem of synthesizing output-feedback control laws for a class of discrete-time hybrid systems in order for the trajectories of the system to satisfy certain high-level specifications expressed in linear temporal logic. By leveraging ideas from robust interpretation of temporal logic formulas and bounded-error estimation, we identify a subclass of systems for which it is possible to reduce the problem to a state-feedback form. In particular, we use locally superstable hybrid observers to resolve the partial information at the continuous level. This allows us to use recent results in temporal logic planning to synthesize the desired controllers based on two-player perfect-information games. The overall control architecture consists of a hybrid observer, a highlevel switching protocol and a low-level continuous controller. We demonstrate the proposed framework in a case study on designing control protocols for an aircraft air management system.
\end{abstract}

\section{INTRODUCTION}

Correct-by-construction controller synthesis for hybrid systems from temporal logic specifications has attracted considerable attention in the past decade. At present, hybrid systems are put to use both in industrial settings and in products such as cars and airplanes [14]. Safety-criticality of such systems creates a need to synthesize controllers that enforce hybrid systems to satisfy certain high-level specifications, e.g., on safety, reliability and performance.

A typical solution for such control synthesis problems is a hierarchical control architecture with several discrete and continuous layers (see, for instance, [9], [7], [10], [20], [11] and references therein). One of the limitations of these approaches is that they rely on availability of the full system state for feedback. However, in many applications of interest, it is not possible to equip the system with a multitude of sensors both for reasons of economy and physical space. Motivated by these limitations, we propose in this paper a framework that can guarantee correctness with limited measurements through output feedback.

Previous work on synthesis with partial state information has mostly focused on the discrete level [3], [15]. Except for some special cases [8], handling the imperfect state information at the discrete level requires a power set construction (i.e., construction of a belief space) that has prohibitive computational complexity. In this paper, we consider partial

\footnotetext{
${ }^{1} \mathrm{O}$. Mickelin is with the Royal Institute of Technology (KTH), Sweden, oscarmi@kth.se.

${ }^{2} \mathrm{~N}$. Ozay is with the Department of Electrical Engineering and Computer Science, University of Michigan, Ann Arbor, necmiye@umich.edu.

${ }^{3}$ R. M. Murray is with the Department of Control and Dynamical Systems, California Institute of Technology, murrayecds.caltech.edu.
}

observability of the continuous state. In order not to experience a complexity blow-up at the discrete level, we deal with the partial state information at the continuous level. We leverage ideas from robust interpretation of temporal logic formulas [6] and bounded-error estimation [13] to develop a framework for synthesizing provably-correct output-feedback control laws for discrete-time piecewise-affine systems. In particular, for a class of systems admitting locally superstable hybrid observers [4], [2], we show that the problem can be reduced to a state-feedback form, which can then be solved using available tools [21].

The rest of the paper is organized as follows. Section III presents some background results. The problem is formally stated and an overview of the solution strategy is given in section III. The main results are presented in sections IV V Section VI demonstrates an application of the proposed framework to a case study on aircraft air management systems. Finally, section VII concludes the paper with some remarks.

\section{Preliminaries}

\section{A. Notation}

All vector and matrix norms considered in this article will be the infinity norms, denoted without subscripts. Therefore, given a matrix $A=(A)_{i j}$ and a vector $x=\left[x_{1}, \ldots, x_{n}\right]^{T}$, we define $\|A\|=\max _{i} \sum_{j}\left|A_{i j}\right|,\|x\|=\max _{i}\left|x_{i}\right|$. The $i^{t h}$ row vector of $A$ is written as $[A]_{i}$. The row and column spaces of $A$ is denoted by $\operatorname{row}(A)$ and $\operatorname{col}(A)$, respectively, with $\operatorname{dimensions} \operatorname{dim}(\operatorname{row}(A))$ and $\operatorname{dim}(\operatorname{col}(A))$; and the dimension of the null space is written as nullity $(A)$. The diameter of a set $X \subseteq R^{n}$ is denoted by $\operatorname{diam}(X)$, and its distance from a point $p \in \mathbb{R}^{n}$ by $d(p, X)=\inf _{x \in X}\|p-x\|$.

With a point $p \in \mathbb{R}^{n}$ and $r \in \mathbb{R}$, we denote the ball centered at $p$ with radius $r$ as $B(p, r)=\left\{x \in \mathbb{R}^{n}: \| x-\right.$ $p \| \leq r\}$. Lastly, given a matrix $H \in \mathbb{R}^{m \times n}$ and a vector $k \in \mathbb{R}^{m}$, a polytope is a set $P=\{x: H x \leq k\} \subseteq \mathbb{R}^{n}$, where the inequality is interpreted element-wise, i.e., $P=$ $\left\{x:[H x]_{i} \leq[k]_{i}, i=1, \ldots, m\right\}$.

Given a set $Q, Q^{\omega}\left(Q^{+}\right)$denotes the set of infinite (nonempty finite) sequences of elements in $Q$.

\section{B. System and environment models}

We consider discrete-time piecewise affine systems formally defined as follows.

Definition 1: A discrete-time piecewise-affine system is a tuple $S=\left(X,\left\{R_{k}\right\}_{k=1}^{k_{\max }},\left\{\mathfrak{D}_{k}\right\}_{k=1}^{k_{\max }}\right)$ where:

- $X \subseteq \mathbb{R}^{n}$ is a compact set called the state space. 
- The regions $R_{k} \subseteq X, R_{i} \cap R_{j}=\emptyset$ for $i \neq j$, form a partition of $X$.

- $\mathfrak{D}_{k}$ is the dynamics in region $R_{k}$, that is the state $x$ evolves with

$$
\begin{aligned}
x(t+1) & =A_{k} x(t)+B_{k} u(t)+F_{k}+E_{k} \delta(t) \\
y(t) & =C_{k} x(t)
\end{aligned}
$$

when $x(t) \in R_{k} \subseteq X$. In Eq. (1), $y(t) \in \mathbb{R}^{l}$ is the measured output, $u(t) \in U \subseteq \mathbb{R}^{m}$ is the control input, $x(t) \in \mathbb{R}^{n}$ is the state variable, and $\delta(t) \in W \subseteq \mathbb{R}^{d}$ is the disturbance. $A_{k} \in \mathbb{R}^{n \times n}, B_{k} \in \mathbb{R}^{n \times m}, C_{k} \in \mathbb{R}^{n \times l}$, $F_{k} \in \mathbb{R}^{n \times 1}$ and $E_{k} \in \mathbb{R}^{n \times d}$ are the system matrices corresponding to region $R_{k}$.

We let $Y$ denote the set of outputs, that is, $Y \doteq\{C x$ : $x \in X\}$. Given a system $S$, a subset $X^{\prime} \subseteq X$ is said to respect the dynamics if $X^{\prime} \cap R_{k} \neq \emptyset$ only for a unique $k$.

In addition to the disturbances in the system model, we consider an external environment to refer to the "discrete" factors that are relevant to the operation of the system, but do not impact its dynamics directly, i.e., not explicitly appear in Eq. (1). Since such factors are not necessarily controlled by the system, e.g., traffic lights, weather conditions, user inputs etc., they are treated as adversaries. We use a simple transition system to model environment evolution.

Definition 2: An environment model is a tuple $\mathcal{T}_{e}=$ $\left(\mathcal{E}, \mathcal{E}_{0}, \rightarrow\right)$ where:

- $\mathcal{E}$ is a finite set of states.

- $\mathcal{E}_{0} \subseteq \mathcal{E}$ is a set of initial states, i.e., $e(0) \in \mathcal{E}_{0}$.

- $\rightarrow \subseteq \mathcal{E} \times \mathcal{E}$ is a transition relation that governs the evolution of the environment. That is, $(e(t), e(t+1)) \in \rightarrow$ for all $t \geq 0$.

The discrete environment is assumed to be fully observable by the system.

Remark 1: For the clarity of the presentation, we restrict the system dynamics to the form in Eq. (1). Our framework can be easily extended to cases where there is measurement noise or where the dynamics include controllable and uncontrollable switches (e.g., using ideas from [16], [11]). Also, our framework allows general $U, W$ and $\left\{R_{k}\right\}_{k=1}^{k_{\max }}$ sets, but, in what follows, we assume these sets are bounded convex polytopes to facilitate computation.

\section{Linear temporal logic and protocols}

Linear temporal logic (LTL) is a formal language that extends the standard propositional logic with temporal operators to express complex, temporal tasks [1]. LTL has proven useful in e.g., software and hardware verification, robotics and other applications of control synthesis, allowing for succinct and expressive specification of system behavior.

1) Syntax and semantics: Before defining the syntax and semantics of LTL, we need a few definitions. A combined state of the system and the environment is a tuple $s(t) \doteq$ $(e(t), x(t)) \in \mathcal{E} \times X$ and a trajectory is an infinite sequence of states of the form $s=s(0) s(1) \ldots \in(\mathcal{E} \times X)^{\omega}$. An atomic proposition is a function from the set of states to boolean true and false. We denote the set of all atomic propositions by $\Pi$. In our context each $\pi_{i} \in \Pi$ is an indicator function of a set $\llbracket \pi_{i} \rrbracket=\{e\} \times X_{i}$, with $e \in \mathcal{E}$ and $X_{i} \subseteq X$ is a convex polytope, wherein $\pi$ evaluates to true. A set $X^{\prime} \subseteq X$ is said to respect the propositions if for all $x \in X^{\prime}$ the same set of propositions hold.

The syntax of an LTL formula over a set of atomic propositions $\Pi$ is given by the following grammar:

$$
\varphi::=\operatorname{true}|\pi| \varphi_{1} \wedge \varphi_{2}|\neg \varphi| \bigcirc \varphi \mid \varphi_{1} \mathcal{U} \varphi_{2},
$$

where $\pi \in \Pi$, and $\varphi_{1}$ and $\varphi_{2}$ are LTL formulas. The symbols $\wedge, \neg, \bigcirc$ and $\mathcal{U}$ stand for the logical operators conjunction, negation and temporal operators next and until, respectively. These operators can be used to define additional operators such as disjunction $(\vee)$, implication $(\rightarrow)$, always $(\square)$ and eventually $(\diamond)$. We will consider specifications in an assume/guarantee form

$$
\varphi \doteq \varphi_{e} \rightarrow \varphi_{s},
$$

where $\varphi_{e}$ encodes assumptions on the environment and $\varphi_{s}$ specifications of the desired system behavior.

Satisfaction of a formula $\varphi$ at a state $s(t)$ in a trajectory $s$ is denoted by $s(t) \models \varphi$ and is defined by letting

1) $s(t)=$ true;

2) For any atomic proposition $\pi, s(t) \models \pi$ if $s(t) \in \llbracket \pi \rrbracket$;

3) $s(t) \models \varphi_{1} \wedge \varphi_{2}$ if $s(t) \models \varphi_{1}$ and $s(t) \models \varphi_{2}$;

4) $s(t) \models \neg \varphi$ if $s(t) \not \models \varphi$;

5) $s(t) \models \bigcirc \varphi$ if $s(t+1) \models \varphi$;

6) $s(t) \models \varphi_{1} \mathcal{U} \varphi_{2}$ if $\exists j \in \mathbb{N}$ s.t. $s(i) \models \varphi_{1}, s(j) \models \varphi_{2}$, $\forall i \in[t, j)$.

The trajectory $s$ satisfies the formula $\varphi$ if $s(0) \models \varphi$. We say that a formula $\varphi$ of the form (2) is satisfied by the system if it is satisfied by all possible trajectories of the system which are consistent with the dynamics in (1) and for all environment behaviors captured by the environment model.

A state-feedback control protocol $\mathcal{C}$ is a partial function on non-empty sequences of states of the system with

$$
\begin{aligned}
\mathcal{C}:(\mathcal{E} \times X)^{+} \times \mathcal{E} & \rightarrow U \\
(s(0), s(1) \ldots, s(t-1), e(t)) & \mapsto u(t)
\end{aligned}
$$

where $u(t)$ is the input signal to be used in the subsequent time-step. Lastly, by letting $r(t) \doteq(y(t), e(t))$, we define an output-feedback control protocol as

$$
\begin{aligned}
\mathcal{C}:(\mathcal{E} \times Y)^{+} \times \mathcal{E} & \rightarrow U \\
(r(0), r(1) \ldots, r(t-1), e(t)) & \mapsto u(t)
\end{aligned}
$$

where $u(t)$ is decided upon by only using the measured output.

2) Robust satisfaction of LTL formulas: Following [6], this section describes a robust interpretation of LTL formulas. Given $\pi \in \Pi$, define $\pi^{\varepsilon}$, by $\llbracket \pi^{\varepsilon} \rrbracket=\left\{(e, x) \in \llbracket \pi \rrbracket:\left(e, x^{\prime}\right) \in\right.$ $\left.\llbracket \pi \rrbracket, \forall x^{\prime} \in B(x, \varepsilon)\right\} . \llbracket \pi \rrbracket$ denotes a robust version of the atomic proposition $\llbracket \pi \rrbracket$, which will be used in connection with estimation errors below. We can extend the robustness properties to general LTL formulas as follows. Take $\varphi$ as a formula written on Negation Normal Form [5]. Form $\neg \Pi=$ $\{\neg \pi: \pi \in \Pi\}$ and let $\hat{\Pi}=\Pi \cup \neg \Pi$. Now, interpreting $\varphi$ as a formula over $\hat{\Pi}$, replace all atomic propositions $\pi$ by 
$\pi^{\varepsilon}$ and denote the resulting formula by $\varphi^{\varepsilon}$. We say that a system satisfies a formula $\varphi$ ह-robustly if it satisfies $\varphi^{\varepsilon}$.

\section{PROBlem FORMUlation AND SOLUTION STRATEGY}

Next, we formally state the problem and give an overview of the proposed solution.

Problem 1: Given a system $S=\left(X,\left\{R_{k}\right\}_{k=1}^{k_{\max }}\right.$, $\left.\left\{\mathfrak{D}_{k}\right\}_{k=1}^{k_{\max }}\right)$, an environment $\mathcal{T}_{e}=\left(\mathcal{E}, \mathcal{E}_{0}, \rightarrow\right)$, a set $\Pi$ of propositions together with an LTL formula $\varphi$ as in (2), and a set $X_{0} \subseteq X$ that respects the propositions and dynamics, construct an output feedback control protocol that satisfies $\varphi$ for all initial conditions $x(0)$ in $X_{0}$ and for all possible environment behaviors in $\mathcal{T}_{e}$ using only the measured output $y$.

We denote an instance of Problem 1 as a tuple $\left(S, X_{0}, \mathcal{T}_{e}, \varphi\right)$. When a solution to a problem $\left(S, X_{0}, \mathcal{T}_{e}, \varphi\right)$ exists, we say the problem is realizable.

Starting from a system model given in the form of Def. 1 and an LTL specification in the form of (2), we use an approach centered on observer estimations of the state space in order to solve Problem 1 1 The proposed framework exploits the hierarchical approach considered in earlier work [20], [16] and consists of the following steps:

1) Find a locally superstable observer with an appropriate equalized performance level and redefine the system dynamics and LTL specifications based on the estimated state.

2) Produce a discrete abstraction based on the redefined dynamics.

3) Use existing techniques in automata theory to design a control protocol guaranteeing correctness of the system.

4) Implement the automaton for continuous execution by combining the observer with low-level controllers.

In section $I V$ we discuss certain types of observers that are suitable for steps 1 and 4 . In section $\mathrm{V}$, we prove that given such an observer, one can still guarantee correctness when using the redefined dynamics in steps 2 and 3 and treating the problem as a state-feedback problem as in [20], [16]. We also briefly overview the results from [20], [16] necessary to complete these design steps.

The overall control architecture shown in Fig. 1 consists of a hybrid observer, a high-level switching protocol and a low-level continuous controller.

\section{OBSERVER DESIGN}

In order to utilize possible partially known state information, we use observers

$$
\begin{aligned}
\mathcal{O}:(Y \times U)^{*} & \rightarrow X \\
(p(0), p(1) \ldots, p(t-1)) & \mapsto \hat{x}(t)
\end{aligned}
$$

where $p(t) \doteq(y(t), u(t))$ and $\hat{x}(t)$ is an estimate of the state. The estimation error at time $t$ is denoted by $\xi(t) \doteq$ $x(t)-\hat{x}(t)$. The design of an observer is made more difficult for piecewise-affine systems as an error in the estimate ambiguities the underlying dynamics. Moreover, any atomic proposition $\pi \in \Pi$ holds true in a limited region $\llbracket \pi \rrbracket \subseteq$

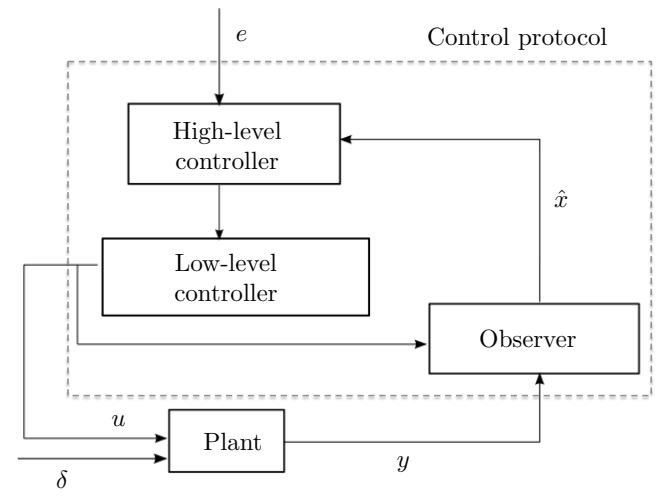

Fig. 1. The proposed control architecture.

$\mathcal{E} \times X$; and $(e, \hat{x}) \in \llbracket \pi \rrbracket$ does not imply $(e, x) \in \llbracket \pi \rrbracket$. Therefore, upper bounds on the estimation errors are needed.

Typically, optimal observers that minimize the estimation error when there are persistent disturbances can be arbitrarily complex even for linear systems [18], [13]. Instead of seeking optimal bounds, we adopt the notion of equalized performance from [2] to characterize observers.

Definition 3: [Equalized performance] An observer is said to achieve an equalized performance level $\mu$ if, whenever $\|\xi(t)\| \leq \mu$, we have $\|\xi(t+1)\| \leq \mu$.

For a piecewise-affine system as in Def. 11, we consider fixed-complexity locally-affine observers of the form

$$
\hat{x}(t+1)=\left(A_{k}-L_{k} C_{k}\right) \hat{x}(t)+B_{k} u(t)+F_{k}+L_{k} y(t),
$$

with a collection of linear filter gains $L_{k} \in \mathbb{R}^{n \times l}$, one for each $R_{k}$.

Proposition 1: Consider an observer of the form (6). Assume, for the time being, that the observer has perfect knowledge of $k$ (i.e., the region $R_{k}$ the true state $x(t)$ is in) at all times Then, choosing the filter gains $L_{k}$ in Eq. (6) such that

$$
\left\|A_{k}-L_{k} C_{k}\right\| \leq 1-\frac{\max _{\delta \in W}\left\|E_{k} \delta\right\|}{\varepsilon}
$$

for all $k$ leads to an equalized performance level $\varepsilon$.

Proof: Since $k$ is known by assumption, the estimation error evolves as

$$
\xi(t+1)=\left(A_{k}-L_{k} C_{k}\right) \xi(t)+E_{k} \delta(t) .
$$

By equating the norms of both sides of Eq. (8) and with simple manipulation, one can see that if $\|\xi(t)\| \leq \varepsilon$ and Eq. (7) holds, we have $\|\xi(t+1)\| \leq \varepsilon$.

Definition 4: [Locally detectable system] An observer of the form (6) that satisfies Eq. (7) is called a locally superstable observer with equalized performance $\varepsilon$. A piecewise affine system is called locally detectable with performance level $\varepsilon$ if it admits a locally superstable observer with equalized performance $\varepsilon$.

\footnotetext{
${ }^{1}$ Note that this is true at $t=0$ because $X_{0}$ respects the dynamics. In section $\mathrm{V}$ we show how to synthesize the control protocol so that it chooses the consequent control inputs $u$ to ensure this at later time steps.
} 
Note that this condition is more restrictive than the notion of detectability as, firstly, detectability only concerns the behavior of a trajectory as time goes to infinity, and secondly, the existence of a matrix $L$ such that $\|A-L C\|<1$ as in Eq. (7) is a sufficient condition for detectability of $(A, C)$.

Finally note that checking if a system is locally superstable with a given performance level and if so, finding the corresponding filter gains, can be efficiently performed via linear programming.

\section{A. Conditions on system matrices for superstability}

In this section, we present an alternative characterization of a subclass of systems for which there exist locally superstable observers with equalized performance $\varepsilon$. These characterizations can provide guidelines for designing the measurement matrices $C_{k}$, when sensor selection is part of the design. Proofs of the following propositions can be found in [12].

Proposition 2: There exists a family of filter gains $L_{k}$ for a system of the form (1) with $\left\|A_{k}-L_{k} C_{k}\right\| \leq \varepsilon$ if and only if for each $k$, there exists a $D_{k} \in \mathbb{R}^{n \times n}$ with

1) $\operatorname{row}\left(A_{k}+D_{k}\right) \subseteq \operatorname{row}\left(C_{k}\right)$

2) $\left\|D_{k}\right\|<\varepsilon$.

Note that as $\operatorname{dim}\left(\operatorname{col}\left(C_{k}^{T}\right)\right) \leq l$, a filter achieving the required bound is more easily constructed when $A_{k}+D_{k}$ has low rank or when $l \rightarrow n$, which intuitively corresponds to the two cases where either $A_{k}+D_{k}$ does not hold much information or when $C_{k}$ gives practically full state information, respectively.

Proposition 3: If there exists a family of filter gains $L_{k}$ for a system of the form (1) with $\left\|A_{k}-L_{k} C_{k}\right\| \leq \varepsilon$, then for each $k$, there exists a $D_{k} \in \mathbb{R}^{n \times n}$ with

1) $\operatorname{nullity}\left(A_{k}^{T}+D_{k}^{T}\right) \geq n-l$

2) $\left\|D_{k}\right\|<\varepsilon$.

Note that this sufficiently characterizes the systems for which the developed framework is possible to use.

\section{REDUCTION TO STATE FEEDBACK}

Obviously, if we can ensure that the estimated state trajectory robustly satisfies an LTL formula and the estimation error can be kept globally bounded, then the true trajectory satisfies the LTL formula. This fact is stated formally next.

Theorem 1: Let $s=s(0) s(1) \ldots$ be an infinite sequence where $s(t)=(e(t), x(t))$ for all $t$. Similarly, define $\hat{s}=$ $\hat{s}(0) \hat{s}(1) \ldots$ where $\hat{s}(t)=(e(t), \hat{x}(t))$ for all $t$. Given an LTL formula $\varphi$, if there exists a bound $\varepsilon \geq 0$ such that $\hat{s}=\varphi^{\varepsilon}$ and $\|\hat{x}(t)-x(t)\| \leq \varepsilon$ for all $t$, then $s=\varphi$.

Proof: Follows directly from the definition of robust satisfaction of LTL formulas.

In order to be able to employ this result, we need to establish a global bound $\varepsilon$ on the estimation error using locally superstable observers. We first consider an intermediate result.

Lemma 1: Let $S=\left(X,\left\{R_{k}\right\}_{k=1}^{k_{\max }},\left\{\mathfrak{D}_{k}\right\}_{k=1}^{k_{\max }}\right)$ be a system. If $S$ is locally detectable with performance level $\operatorname{diam}\left(X_{0}\right)$ and $\hat{x}(t) \in \bigcup_{k=1}^{k_{\max }} \hat{R}_{k}$ for all $t \geq 0$, where $\hat{R}_{k} \doteq$ $\left\{x \in R_{k}: B\left(x, \operatorname{diam}\left(X_{0}\right)\right) \subseteq R_{k}\right\}$ then $\|\xi(t)\| \leq \operatorname{diam}\left(X_{0}\right)$ for all $t \geq 02$

Proof: Since $S$ is locally detectable with performance level $\operatorname{diam}\left(X_{0}\right)$, there exist $L_{k}$ such that Eq. (7) holds. By Proposition 1, the estimation error can be bounded if the unique region $R_{k}$ with $x \in R_{k}$ is known. By the assumptions in problem formulation 1 , this is known at $t=0$ and we proceed by induction on $t$. Given $x(t) \in R_{k}$, we know $\|\xi(t)\| \leq \operatorname{diam}\left(X_{0}\right)$. By the definition of the shrunk regions, $d\left(x(t), \hat{R}_{j}\right)>\operatorname{diam}\left(X_{0}\right)$, for $j \neq k$ so if $\hat{x}(t) \notin \hat{R}_{k}$, then $\|\xi(t)\|=\|x(t)-\hat{x}(t)\| \geq d\left(x(t), \hat{R}_{j}\right)>\operatorname{diam}\left(X_{0}\right)$, which contradicts the induction hypothesis. Therefore, $\hat{x}(t) \in \hat{R}_{k}$ and, in the next time step, we can measure $\hat{x}(t+1) \in \hat{R}_{j}$, for some $1 \leq j \leq k_{\max }$ and obtain $\|\xi(t+1)\| \leq \operatorname{diam}\left(X_{0}\right)$, by Proposition 1 . This concludes the induction step and the proof is done.

We associate each locally detectable system with another system whose outputs are equal to its states.

Definition 5: Given a locally detectable system $S=$ $\left(X,\left\{R_{k}\right\}_{k=1}^{k_{\max }},\left\{\mathfrak{D}_{k}\right\}_{k=1}^{k_{\max }}\right)$ that admits a locally superstable observer with performance level $\varepsilon$ and corresponding gains $L_{k}$, the $\varepsilon$-robust observer system $\hat{S}=\left(\hat{X},\left\{\hat{R}_{k}\right\}_{k=1}^{k_{\max }}\right.$, $\left.\left\{\hat{\mathfrak{D}}_{k}\right\}_{k=1}^{k_{\max }}\right)$ is given by the following parameters:

- $\hat{X}=\bigcup_{k=1}^{k_{\max }} \hat{R}_{k}$,

- $\hat{R}_{k}=\left\{x \in R_{k}: B(x, \varepsilon) \subseteq R_{k}\right\}$,

- $\hat{\mathfrak{D}}_{k}$ is the dynamics in region $\hat{R}_{k}$, with

$$
\begin{aligned}
\hat{x}(t+1) & =\hat{A}_{k} \hat{x}(t)+\hat{B}_{k} \hat{u}(t)+\hat{F}_{k}+\hat{E}_{k} \hat{\delta}(t) \\
\hat{y}(t) & =\hat{x}(t),
\end{aligned}
$$

where $\hat{A}_{k}=A_{k}, \hat{B}_{k}=B_{k}, \hat{F}_{k}=F_{k}, \hat{E}_{k}=L_{k} C_{k}$, $\hat{u}(t) \in \hat{U}=U$ and $\hat{\delta}(t) \in \hat{W}=B(0, \varepsilon)$.

An observer for $S$ as in Eq. (6) is said to be consistent with an $\varepsilon$-robust observer system $S$ if they use the same gains $L_{k}$.

Now we state the main result of this section where $\varepsilon$ robust observer systems are used to pose an alternative perfect-information problem, the solution of which provides a solution to Problem 1,

Theorem 2: Define $\varepsilon^{\prime} \doteq \operatorname{diam}\left(X_{0}\right)$. Given an instance $\left(S, X_{0}, \mathcal{T}_{e}, \varphi\right)$ of Problem 1] assume $S$ is locally detectable with performance level $\varepsilon^{\prime}$. Let $\hat{S}=\left(\hat{X},\left\{\hat{R}_{k}\right\}_{k=1}^{k_{\max }}\right.$, $\left\{\hat{\mathfrak{D}}_{k}\right\}_{k=1}^{k_{\max }}$ ) be a $\varepsilon^{\prime}$-robust observer system for $S$. Then, if there exists a state-feedback control protocol for $\hat{S}$ that makes the system satisfy $\varphi^{\varepsilon^{\prime}}$ for some initial condition $\hat{x}(0) \in X_{0} \cap \hat{X}$ and for all possible environment behaviors in $\mathcal{T}_{e},\left(S, X_{0}, \mathcal{T}_{e}, \varphi\right)$ is realizable. Moreover, an outputfeedback protocol for $S$ can be constructed by using an observer consistent with the dynamics of $\hat{S}$ and by driving the system $S$ with the same control signals as applied to $\hat{S}$.

Proof: By definition, the state-feedback control protocol for $\hat{S}$ that makes the system satisfy $\varphi^{\varepsilon^{\prime}}$ ensures that $\hat{x}(t) \in \hat{X}$ for all $t$, i.e., the states remain within the statespace of the system. Therefore, by Lemma 1 , the estimation

\footnotetext{
${ }^{2}$ To be precise, a performance level strictly less than $\operatorname{diam}\left(X_{0}\right)$ is required to accommodate trajectories with $x(t)$ on a border between two regions. However, such cases are negligible from a practical standpoint and will be disregarded.
} 
error can be globally bounded by $\varepsilon^{\prime}$ for any initial condition $\hat{x}(0) \in X_{0} \cap \hat{X}$ while running the system $S$ and the observer with the input signal from this protocol. Finally, invoking Theorem 1 concludes the proof.

\section{A. Overview of full-information synthesis}

This section briefly describes the process of obtaining state-feedback control protocols. The full details can be found in e.g., [16], [20].

Based on previous work [16], [20], a discrete synthesis procedure can be phrased as a two-player perfect information game, where the environment is treated as an adversary, i.e., it is assumed to make the worst-case transitions consistent with its transition relation in Def. 2 and the assumption $\varphi_{e}$ part of the specification. In order to incorporate the piecewise affine system in this game, constructing a finite transition system representing the dynamics is required [20]. This construction relies on partitioning the continuous statespace to create discrete-states and solving short-horizon constrained reachability problems between regions to establish the transition relations.

In order to solve the state-feedback synthesis problem stated in Theorem 2, we create a discrete-transition system for the $\varepsilon$-robust observer system, where the reachability computations are performed on shrunk regions. The first step in doing so produces a proposition preserving partition $X=\bigcup_{i=1}^{n} P_{i}$ respecting the system dynamics. Assuming $P_{i}$ to be a convex polytope defined by $H_{i} x \leq k_{i}$, a shrunk polytope can then be defined as

$$
\hat{H}_{i} x \leq \hat{k}_{i}
$$

where $\hat{H}_{i}=H_{i}$ and $\left[\hat{k}_{i}\right]_{j}=\left[k_{i}\right]_{j}-\varepsilon\left\|\left[H_{i}\right]_{j}\right\|$. This gives the following result.

Proposition 4: If $\|\xi(t)\| \leq \varepsilon$ and $\hat{x}(t) \in \hat{P}_{i}$, then $x(t) \in$ $P_{i}$.

Proof: We have $H_{i} x(t)=H_{i}(x(t)-\hat{x}(t)+\hat{x}(t))=$ $H_{i}(x(t)-\hat{x}(t))+\hat{H}_{i} \hat{x}(t)$. In the last equality, $\| x(t)-$ $\hat{x(t)} \| \leq \varepsilon$, and by construction, $\left[\hat{H}_{i} \hat{x}(t)\right]_{j} \leq\left[k_{i}\right]_{j}-\varepsilon\left\|\left[H_{i}\right]_{j}\right\|$, so we obtain $\left[H_{i} x(t)\right]_{j} \leq\left[\hat{H}_{i} \hat{x}(t)\right]_{j}+\varepsilon\left\|\left[H_{i}\right]_{j}\right\| \leq\left[k_{i}\right]_{i}-$ $\varepsilon\left\|\left[H_{i}\right]_{i}\right\|+\varepsilon\left\|\left[H_{i}\right]_{j}\right\|=\left[k_{i}\right]_{j}$.

Now, appealing to Theorem 2, transitions for the estimated state $\hat{x}$ between regions $\hat{P}_{i}$ and $\hat{P}_{j}$ lead to transitions of the actual state between regions $P_{i}$ and $P_{j}$, meaning that algorithms for control synthesis [21], [17] applied directly to the estimated system in Definition 5 lead to control protocols of the original system in Problem 1]

Lastly, note that if a region $P_{i}$ in Proposition 4 is given by a union of several convex polytopes, shrinking each of these will yield a valid, although conservative, region for control synthesis.

We summarize the ideas above in the following algorithm:

1) Establish a proposition preserving partition $X=$ $\bigcup_{i=1}^{n} P_{i}$ of the state space domain $X$, respecting the system dynamics.

2) Shrink each region $P_{i}$. Establish transition relations for each of the new polytopes $\hat{P}_{i}$.

3 ) Force $\hat{x}$ to transition between the shrunk polytopes.

\section{CASE STUdY: AIR MANAGEMENT SYSTEM OF AIRCRAFT}

This section uses a simplified and linearized model of an air management system (AMS) of an aircraft as a test-case for the theory developed in sections II V above.

A conventional AMS operates by admitting ambient air into the engines of an aircraft and forwarding this to a so called pressurization and air conditioning kit, where pressure is controlled by electrical compressors, temperature by a heat exchanger (HX) and possibly expansion cooling in a turbine, and finally humidity by a high pressure water extraction loop [14]. The AMS needs to be designed so as to supply sufficient pressure to the cabin at bearable temperature and humidity, preferably under comfortable conditions. It is also responsible for providing the cabin with its supply of fresh oxygen. Restrictions in the amount of power that can be supplied to the electronics and sensitivity of sensors to e.g, high temperature, exist; also, freezing of different parts of the craft pose operational problems. Lastly, the AMS should be fault tolerant as it is a critical part of the craft.

TABLE I

THE SYMBOLS USED IN THE SIMPLIFIED AMS MODEL.

\begin{tabular}{|c|c|c|}
\hline Symbol & Unit & Description \\
\hline \multicolumn{3}{|c|}{ Measurable states } \\
\hline$T_{x}$ & ${ }^{\circ} \mathrm{C}$ & Temperature of metal in heat exchanger \\
\hline$T_{c}$ & ${ }^{\circ} \mathrm{C}$ & Temperature of cabin \\
\hline \multicolumn{3}{|c|}{ Non-measurable state } \\
\hline$p_{v}$ & $\mathrm{kPa}$ & Outlet air pressure of valve 1 \\
\hline \multicolumn{3}{|c|}{ Controllable variables } \\
\hline$C_{1}$ & & Valve coefficient for valve 1 \\
\hline$C_{2}$ & & Valve coefficient for valve 2 \\
\hline$W_{a}$ & $\mathrm{~kg} / \mathrm{s}$ & Mass flow rate of cold inflow in HX \\
\hline \multicolumn{3}{|c|}{ Switched variables } \\
\hline$T_{a}$ & $\mathrm{~K}$ & Temperature of cold inflow in HX (ambient air) \\
\hline$T_{e}$ & $\mathrm{~K}$ & Temperature of the air from the engine \\
\hline \multicolumn{3}{|c|}{ Other derived variables } \\
\hline$W_{i}$ & $\mathrm{~kg} / \mathrm{s}$ & Incoming mass flow rate of the air from the engine \\
\hline$W_{v}$ & $\mathrm{~kg} / \mathrm{s}$ & Mass flow rate of the air that goes through valve 2 \\
\hline$W_{h}$ & $\mathrm{~kg} / \mathrm{s}$ & Mass flow rate of the air that goes through the $\mathrm{HX}$ \\
\hline$T_{h}$ & $\mathrm{~K}$ & Outlet air temperature of the HX \\
\hline \multicolumn{3}{|c|}{ Constant variables } \\
\hline$p_{e}$ & $\mathrm{kPa}$ & Pressure of the air from the engine \\
\hline$p_{c}$ & $\mathrm{kPa}$ & Pressure of the cabin \\
\hline$W_{f}$ & $\mathrm{~kg} / \mathrm{s}$ & Mass flow rate passing through the fan \\
\hline
\end{tabular}

TABLE II

NUMERICAL VALUES USED IN THE SIMPLIFIED AMS MODEL

\begin{tabular}{c|c|c}
\hline Symbol & Value & Description \\
\hline \hline Measurable states & & \\
\hline$T_{x}$ & $297.2 \mathrm{~K}$ & Equilibrium value \\
$T_{c}$ & $268 \mathrm{~K}$ & Equilibrium value \\
\hline \hline Non-measurable state & & \\
\hline$p_{v}$ & $136791 \mathrm{~Pa}$ & Equilibrium value \\
\hline \hline Controllable variables & & \\
\hline$C_{1}$ & 0.155 & Equilibrium value \\
$C_{2}$ & 0.18 & Equilibrium value \\
$W_{a}$ & $2.49 \mathrm{~kg} / \mathrm{s}$ & Equilibrium value \\
\hline \hline Switched variables & & \\
\hline$T_{a}$ & $-39,161{ }^{\circ} \mathrm{C}$ & Arbitrary value \\
$T_{e}$ & $207,25^{\circ} \mathrm{C}$ & Arbitrary value \\
\hline \hline Constant variables & & \\
\hline$p_{e}$ & $275.790 \mathrm{kPa}$ & Arbitrary value \\
$p_{c}$ & $101.325 \mathrm{kPa}$ & Arbitrary value \\
\hline
\end{tabular}

A simplified schematic of an AMS is included in Figure VI and the details of the model can be seen in [12]. The symbols used in this section are given in Table I with numerical 


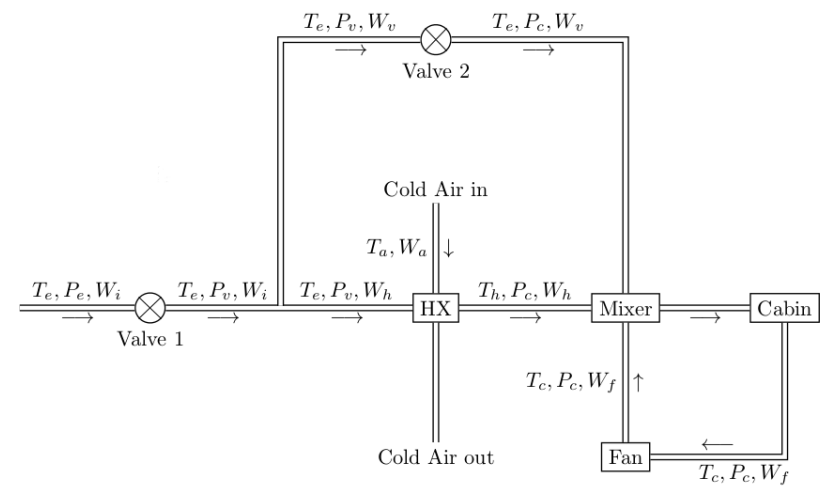

Fig. 2. A simplified AMS model. Variables in this figure are defined in Table [

values listed in Table II The units of the parameters are suppressed in the text below. We introduce switching to the system by assuming the engine temperature to toggle uncontrollably between $T_{e}=207$ and $T_{e}=25$. Also, the airplane can switch its dynamics by having ambient air at either $T_{a}=-39$ or heated to $T_{a}=161$. The uncontrollable switches are assumed to have a time scale larger than the sampling time of the controller, and is here set to 0.5 seconds.

We consider the state space $X=\left\{\left[T_{c}, T_{x}, p_{v}\right]^{T} \in \mathbb{R}^{3}\right.$ : $\left.13 \leq T_{c} \leq 33,-25 \leq T_{x} \leq 15,101.325 \leq p_{v} \leq 275.790\right\}$. Due to the non-linear dynamics of the system, the model results in a set of piecewise affine and linearized dynamics, with three different regions of definition, for every choice of the two environmental and controllable switching modes. These are determined by $R_{1}=\left\{\left[T_{c}, T_{x}, p_{v}\right]^{T} \in X\right.$ : $\left.101.325 \leq p_{v} \leq 137.895\right\}, R_{2}=\left\{\left[T_{c}, T_{x}, p_{v}\right]^{T} \in X:\right.$ $\left.137.895 \leq p_{v} \leq 202.65\right\}$ and $R_{3}=\left\{\left[T_{c}, T_{x}, p_{v}\right]^{T} \in X\right.$ : $\left.202.65 \leq p_{v} \leq 275.790\right\}$, respectively. The control inputs are given by $U=\left\{\left[C_{1}, C_{2}, W_{a}\right]^{T} \subseteq \mathbb{R}^{3}: 0 \leq C_{1}, C_{2} \leq\right.$ $\left.1,0 \leq W_{a} \leq 8.316\right\}$. Lastly, in order to obtain interesting results with limited hardware, the $B$-matrices obtained are amplified by a factor of 7.5. In all, this gives a discrete-time switched piecewise affine system with dynamics of the form

$$
\begin{aligned}
{\left[\begin{array}{l}
T_{c}(t+\Delta t) \\
T_{x}(t+\Delta t) \\
p_{v}(t+\Delta t)
\end{array}\right]=} & A_{k}\left[\begin{array}{l}
T_{c}(t) \\
T_{x}(t) \\
p_{v}(t)
\end{array}\right]+B_{k}\left[\begin{array}{c}
C_{1}(t) \\
C_{2}(t) \\
W_{a}(t)
\end{array}\right]+ \\
& +F_{k}+E_{k} \delta(t) \\
y(t)= & {\left[\begin{array}{lll}
1 & 0 & 0 \\
0 & 1 & 0
\end{array}\right]\left[\begin{array}{l}
T_{c}(t) \\
T_{x}(t) \\
p_{v}(t)
\end{array}\right], }
\end{aligned}
$$

for $k=1,2,3$. We use a sampling time $\Delta t=0.1$ seconds.

\section{A. Specifications}

We assume the cabin crew to be able to set the reference values of $T_{c}$ to hot $\left(T_{c} \in I_{1}=[23.5,25]\right)$, cold $\left(T_{c} \in\right.$ $\left.I_{2}=[21,22.5]\right)$ or intermediate $\left(T_{c} \in I_{3}=[22.5,23.5]\right)$. Cabin crew input is treated as the environment $\mathcal{E}$. The system should eventually reach the reference levels and stay within these levels until told otherwise and we require the environment to not change the reference value until the reference interval has been reached. Also, the cabin temperature should always stay within the temperature range $T_{c} \in[21,25]$. Lastly, we require to always have non-freezing heat exchanger temperature in order to prevent freezing. In order to phrase this in LTL, we represent the cabin crew reference value by a level variable $l \in\{1,2,3\}$ which corresponds to when the reference value is hot, cold and intermediate, respectively. We also introduce a timer $t \in$ $\{0,1, \ldots, 5\}$ and require the reference values to be constant when $t \neq 5$ in order to increase the time scale of the reference value change. The specifications then become:

$$
\begin{aligned}
\varphi_{e} & \rightarrow \varphi_{s}, \\
\varphi_{e} & =\left(\bigwedge_{i=1}^{3} \square\left(\left(l=i \wedge T_{c} \in I_{i}\right) \rightarrow \bigcirc(l)=i\right)\right) \wedge \\
& \wedge \square((t \neq 5) \rightarrow(\bigcirc(t)=t+1)) \wedge \\
& \wedge \square((t=5) \rightarrow(\bigcirc(t)=0)) \wedge \\
& \wedge \square((t \neq 5) \rightarrow(\bigcirc(l)=l)), \\
\varphi_{s} & =\left(\bigwedge_{i=1}^{3} \square\left(l=i \rightarrow \diamond T_{c} \in I_{i}\right)\right) \wedge \\
& \wedge\left(\bigwedge_{i=1}^{3} \square\left(\left(l=i \wedge T_{c} \in I_{i}\right) \rightarrow \bigcirc\left(T_{c}\right) \in I_{i}\right)\right) \wedge \\
& \wedge \square \diamond\left(T_{x} \geq 0\right) .
\end{aligned}
$$

The controllers were synthesized using the Temporal Logic Planning (TuLiP) Toolbox [21], which is a software package designed for temporal logic motion planning interfacing with JTLV [17]. The techniques considered in this article are, however, not limited to this particular choice of software.

\section{B. Simulation}

A sample simulation is included in Figures 344 below, where the initial error in $T_{c}$ and $T_{x}$ were 0.0175 and the initial error in $p_{v}$ was 1.75 . The disturbance term was bounded by 0.016 and 1.6 for $T_{c}, T_{x}$ and $p_{v}$, respectively. For these values and the numerical values of the system matrices, Propositions 2 and 3 can be seen to guarantee existence of a locally superstable observer. With the time $t$ in minutes, the simulation runs with the reference temperature set to intermediate for $0 \leq t<4,16 \leq t<20$, hot for $4 \leq t<10$ and cold for $10 \leq t<16$. In the figures, note the reference following of the cabin temperature and that $T_{c}$ and $p_{v}$ always remain within the state space. The error magnitudes never exceed their initial values, due to local superstability and reduce to the magnitude of the disturbance term during the simulation. Numerically, $\left\|T_{c}(t)-\hat{T}_{c}(t)\right\| \leq$ 0.016, $\left\|T_{x}(t)-\hat{T}_{x}(t)\right\| \leq 0.016,\left\|p_{v}(t)-\hat{p}_{v}(t)\right\| \leq 1.6$ for all times. Note that $T_{c}$ has an error term due to the effect of the disturbance for which the observer cannot compensate between a time step and the next.

\section{CONCLUSIONS AND FUtURE RESEARCH}

In this paper, we described a framework for synthesizing correct-by-construction control protocols for discrete-time 


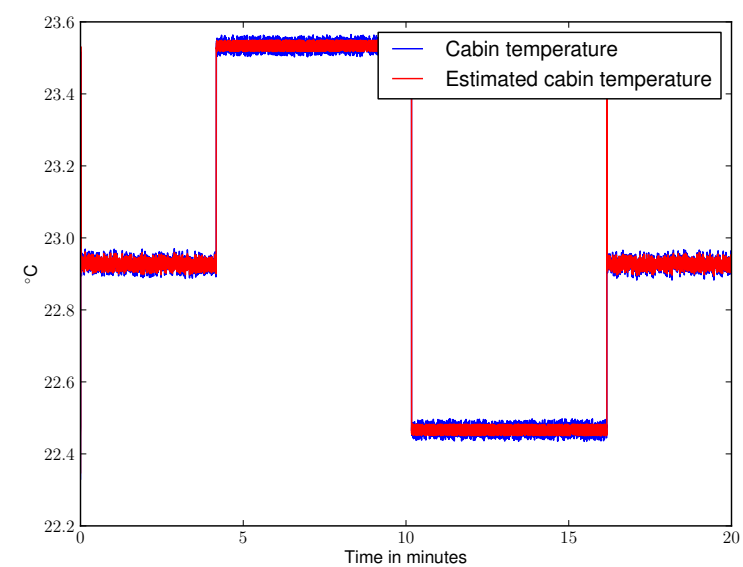

Fig. 3. Cabin temperature for the sample AMS simulation

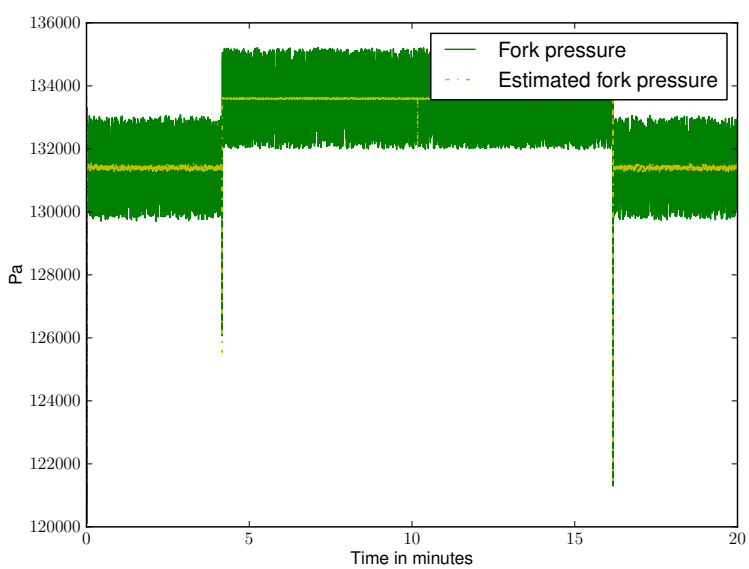

Fig. 4. Pipe fork pressure for the sample AMS simulation

piecewise-affine systems using only partial state information. The main insight of the proposed approach is to resolve the uncertainty in the continuous state at the continuous level of a hierarchical controller so that it is possible to solve a full information problem at discrete level. Ideas from robust estimation were used to design appropriate local observers that are synergistically integrated with the controller stack to achieve global bounds on the estimation errors. The approach was demonstrated on a case-study in the form of an air management system of aircraft.

Future research will consider employing nonlinear or higher-order observers within the proposed framework. Another interesting direction is to investigate whether the relation between the dynamics of a system and the corresponding bounded-error observers can be characterized in terms of alternating approximate simulation relations [19].

\section{ACKNOWLEDGMENT}

This work was supported in part by IBM and UTC through the iCyPhy consortium. The authors wish to thank the iCyPhy team, in particular Yilin Mo from Caltech and Jeff Ernst from UTAS, for helpful discussions on the AMS example and modeling.

\section{REFERENCES}

[1] C. Baier, J.-P. Katoen, et al. Principles of model checking, volume 26202649. MIT press Cambridge, 2008.

[2] F. Blanchini and M. Sznaier. A convex optimization approach to synthesizing bounded complexity $\ell^{\infty}$ filters. Automatic Control, IEEE Trans. on, 57(1):216-221, 2012.

[3] K. Chatterjee, L. Doyen, T.A. Henzinger, and J.-F. Raskin. Algorithms for omega-regular games with imperfect information. Logical Methods in Computer Science, 3(4):1-23, 2007

[4] J. Chen and C.M. Lagoa. Observer design for a class of switched systems. In Decision and Control, 2005 and 2005 European Control Conference. CDC-ECC'05. 44th IEEE Conference on, pages 29452950. IEEE, 2005.

[5] E.M. Clarke, O. Grumberg, and D.A. Peled. Model checking. MIT press, 1999

[6] G.E. Fainekos, A. Girard, H. Kress-Gazit, and G.J. Pappas. Temporal logic motion planning for dynamic robots. Automatica, 45(2):343352, 2009.

[7] A. Girard and G.J. Pappas. Hierarchical control system design using approximate simulation. Automatica, 45(2):566-571, 2009.

[8] M.R. Hafner and D. Del Vecchio. Computational tools for the safety control of a class of piecewise continuous systems with imperfect information on a partial order. SIAM Journal on Control and Optimization, 49(6):2463-2493, 2011.

[9] M. Kloetzer and C. Belta. A fully automated framework for control of linear systems from temporal logic specifications. Automatic Control, IEEE Trans. on, 53(1):287-297, 2008.

[10] H. Kress-Gazit, G.E. Fainekos, and G.J. Pappas. Temporal-logic-based reactive mission and motion planning. Robotics, IEEE Trans. on, 25(6):1370-1381, 2009.

[11] J. Liu, N. Ozay, U. Topcu, and R.M. Murray. Synthesis of reactive switching protocols from temporal logic specifications. Automatic Control, IEEE Trans. on, 58(7):1771-1785, 2013.

[12] O. Mickelin, N. Ozay, and R.M. Murray. Synthesis of correct-by-construction control protocols for hybrid systems using partial state information. Technical report, http://www.cds.caltech.edu/ murray/papers/mom13-acc.html 2013.

[13] M. Milanese and A. Vicino. Optimal estimation theory for dynamic systems with set membership uncertainty: an overview. Automatica, 27(6):997-1009, 1991

[14] I. Moir and A. Seabridge. Aircraft systems: mechanical, electrical and avionics subsystems integration, volume 21 , pages 1-51,259-296. Wiley. com, 2008.

[15] G.E. Monahan. State of the art-a survey of partially observable markov decision processes: Theory, models, and algorithms. Management Science, 28(1):1-16, 1982.

[16] P. Nilsson, N. Ozay, U. Topcu, and R.M. Murray. Temporal logic control of switched affine systems with an application in fuel balancing. In American Control Conference (ACC), 2012, pages 5302-5309. IEEE, 2012

[17] A. Pnueli, Y. Sa'ar, and L.D. Zuck. Jtlv: A framework for developing verification algorithms. In Computer Aided Verification, pages 171174. Springer, 2010.

[18] J.S. Shamma and K.-Y. Tu. Set-valued observers and optimal disturbance rejection. Automatic Control, IEEE Trans. on, 44(2):253-264, 1999.

[19] P. Tabuada. Verification and control of hybrid systems: a symbolic approach. Springer-Verlag New York Inc, 2009.

[20] T. Wongpiromsarn, U. Topcu, and R.M. Murray. Synthesis of control protocols for autonomous systems. Unmanned Systems, pages 1-19, 2013

[21] T. Wongpiromsarn, U. Topcu, N. Ozay, H. Xu, and R.M. Murray. TuLiP: a software toolbox for receding horizon temporal logic planning. In Proceedings of the 14th international conference on Hybrid systems: computation and control, pages 313-314. ACM, 2011 